\title{
Fish diversity of Lohandra River, Biratnagar, Morang, Eastern Nepal
}

\author{
Jay Narayan Shrestha \\ Department of Zoology, Post Graduate Campus, Biratnagar, Tribhuvan University, Nepal \\ Email: jnshrestha545@gmail.com
}

\begin{abstract}
Nepal has great water resources supporting diverged aquatic flora and fauna, including varieties of freshwater fishes. Lohandra river is one of the major perennial river of Morang district, eastern Nepal, originating from Mahabharat hill. Fishes of the river were collected from September, 2014 to August, 2015 using local gears and cast net with the help of local fishermen and the collected fishes were preserved in $70 \%$ alcohol facing their head downward for taxonomic identification. The present paper deals with 51 fish species belonging to 38 genera, 20 families of 7 orders. The notable ichthyofauna of the river were Clarias batracus, Garra gotyla, Botia lohachata, Chagunius chagunio, Chaca chaca, Acanthocobotis botia, Lepidocephalus guntea. Colisa faciatus, Notopterus notopterus, Cirrhinus reba, C. mrigala, Labeo dero, L. cabasu, Salmostoma bacaila, Barilius bendelisis, B. barna, Crossocheilus latius latius, Mystus species, Ompok bimaculatus, Wallago attu, Chanda nama, Nandus nandus, Anabus testudineus, Channa orientalis, C. marulius, Catla catla, Monopterus cuchia, Clupisoma garua and Psilorhyncus sucatio. As Lohandra river was rich with ichthyofauna, the investigation of ecology and fishes of the river is recommended for the conservation of these fishes.
\end{abstract}

Keywords: Fish fauna, Kerabari, Mahabharat, Ompok bimaculatus.

\section{Introduction}

Fishes, a better source of animal protein, are aquatic vertebrates including hag fish, lampreys and cartilaginous and bony fishes which utilized aquatic resources and are converted into animal protein. It is white meat, which is easily digestible so most of the people in the world use the fishes as their major food. Their production is comparatively high because they do not suffer from the loss of substantial amount of energy to maintain body temperature as compared to terrestrial animals.

Nepal, a landlocked country, located in the south of Himalayas in Asia. The Karnali in the west, the Gandaki in the central and the Koshi in eastern part are three major rivers of the country including more than 6,000 other rivers inhabiting 232 fish species (217 native species and 15 exotic species) (Shrestha, 2008), 108 species in Narayani river (Jha \& Bhujel,2014) and 30 species in Seti Gandaki river (Pokharel, 2012). The Koshi river is rich with aquatic fauna and flora. Shrestha (2008) and Gupta (2015) reported some game fish inhabiting in the river are Bagarius bagarius, Channa marulius, Tor putitora, Tor tor, Chitala (Notopterus) chitala, Wallago attu, Aorichthys seenghala, Neolissochilus (Acrossocheilus) hexagonolepis, Clupisoma gaura, Anguilla bengalensis, Silonia silondia, Amphipnous(Monopterus) cuchia and Notopterus notopterus. 
Lohandra river is one of the perennial river of Morang district, Province No. 1, eastern Nepal. It originates from Bhogateni Village Development Committee lying between Mahabharat and Churia hill. It is formed by joining Dhobi Khola and Turke Khola of eighth Bhogateni VDC, then it takes its course of southwards through Kerabari, Belbari, Mohipur and Sidraha, where it joins Judi Khola forming Lohandra river. After crossing Bhathiganj VDC, the river enters in India where it joins with Kosh river.

As regard to iththyofauna, De Witt (1960) reported a contribution to the ichthyology of Nepal. Shrivastava (1968) gave a taxonomic handbook entitled "Fishes of eastern U.P., India", in which he mentioned a number of Nepalese fishes. Thapa and Rajbanshi (1968) worked on the few hill stream fishes of Nepal. Majumdar et al. (1972) has worked on fish fauna of Nepal. Shrestha (1981) published a book "Fishes of Nepal" describing scientific details of 120 fish species. Edds (1986) listed 107 and 111 fish species from the Royal Chitwan National Park and the Kali Gandaki/Narayani River of Nepal respectively. Shrestha (1990) published his book entitled " Resource ecology of the Himalayan water" reporting 74 fish species from Karnali river, 108 species from Trisuli, 102 species from Narayani and 69 species from Mahakali. He also described the swamp land ecology and fish management and conservation in his book. Shrestha (1994) reported 129 fish species belonging to 66 genera in Nepal. She also mentioned a report on Fishes, Fishing implements and Method practiced in the country. Shrestha (2001) published a paper entitled "Taxonomic Revision of Fishes of Nepal", a taxonomic revision of 186 fish species earlier reported (Shrestha, 1998). Shrestha (2008) published a pioneer book of taxonomy entitled "Ichthyology of Nepal" mentioning 232 fish species from Nepal. The rivers of Nepal are really rich in fish fauna which need to be explored scientifically for further conservation. An attempt, therefore, has been made to conduct a thorough survey of fish resources at Lohandra river.

\section{Materials and Methods}

The fish catching sites in the river were selected and were regularly visited. The study area was throughout the river from joining of Sidraha and Judi Khola (upstream) and Bhathiganj (downstream). Fishes from the river were collected with the help of local fisherman using local made fish gears and nets once usually last three days of every month for one year from September, 2014 to August, 2015. The habitat and color of the fishes were recorded on the spot at the time of collection. Alive fishes were killed in $40 \%$ formalin and then preserved in $70 \%$ alcohol facing their head downward. For large fishes, a longitude incisor was made to protect the gut contents. The taxonomic identification of preserved fishes was done with the help of the methods adopted from Shrivastava (1968), Shrestha (2001) and Shrestha (2008). After that the fishes were kept in separate specimen jars using tags and labels for further classification.

\section{Results and discussion}

The present list of fishes includes 51 species belonging to 38 genera, 20 families of 7 orders (Table 1 and Fig.1). There is every chance of over-lapping in fish habitat, which is difficult to be demarcated sharply. 
Table 1. List of fishes found in Lohandra river.

\begin{tabular}{|c|c|c|c|}
\hline No. & Indigenous species & $\begin{array}{l}\text { Common } \\
\text { name }\end{array}$ & $\begin{array}{l}\text { Conservation } \\
\text { status (IUCN) }\end{array}$ \\
\hline 1. & $\begin{array}{l}\text { ORDER: CLUPEIFORMES; Family: Clupeidae } \\
\text { Gadusia chapra (Hamilton-Bachanan) }\end{array}$ & Suiya & $\mathrm{C}$ \\
\hline 2. & $\begin{array}{l}\text { ORDER: OSTEOGLOSSIFORES; Family: Notopteridae } \\
\text { Notopterus notopterus (Palles) }\end{array}$ & Golhai & UN \\
\hline 3. & $\begin{array}{l}\text { ORDER: CYPRINIFORMES;Family: Cyprinidae } \\
\text { Catla cata (Hamilton-Bachanan) }\end{array}$ & Vakur & $\mathrm{C}$ \\
\hline 4. & Chagunius chagunio (Hamilton-Bachanan) & Gelhari & VU \\
\hline 5. & Cirrhinus mrigala (Hamilton-Bachanan) & Naini & $\mathrm{C}$ \\
\hline 6. & C. reba (Hamilton-Bachanan) & Reba & $\mathrm{UN}$ \\
\hline 7. & Labeo rohita (Hamilton-Bachanan) & Rohu & $\mathrm{C}$ \\
\hline 8. & L. dero (Hamilton-Bachanan) & Gurdi & $\mathrm{C}$ \\
\hline 9. & L. cabasu (Hamilton-Bachanan) & Kalbasu & $\mathrm{C}$ \\
\hline 10. & Puntius ticto (Hamilton-Bachanan) & Tite pothi & UN \\
\hline 11. & P. sarana (Hamilton-Bachanan) & Thub pothi & $\mathrm{UN}$ \\
\hline 12. & P. sophore (Hamilton-Bachanan) & Sidra pothi & $\mathrm{C}$ \\
\hline 13. & P. terio (Hamilton-Bachanan) & Pothi & $\mathrm{UN}$ \\
\hline 14. & Chela labuca (Hamilton-Bachanan) & Chelwa & $\mathrm{C}$ \\
\hline 15. & Salmostoma bacaila (Hamilton-Bachanan) & Chilwa & $\mathrm{C}$ \\
\hline 16. & Amblypharyngodon mola (Hamilton-Bachanan) & Mola & $\mathrm{C}$ \\
\hline 17. & Aspidoparis jaya (Hamilton-Bachanan) & Mara & $\mathrm{C}$ \\
\hline 18. & A. morar (Hamilton-Bachanan) & Morar & $\mathrm{C}$ \\
\hline 19. & Barilius bendelisis (Hamilton-Bachanan) & Faketa & $\mathrm{C}$ \\
\hline 20. & B. barna (Hamilton-Bachanan) & Titarkane & $\mathrm{C}$ \\
\hline 21. & Danio devario (Hamilton-Bachanan) & Bhito & $\mathrm{C}$ \\
\hline 22. & Esomus danricus (Hamilton-Bachanan) & Dedhwa & $\mathrm{C}$ \\
\hline 23. & Crossocheilus latius latius (Hamilton-Bachanan) & \multicolumn{2}{|c|}{ Lohari Buduna UN } \\
\hline 24. & Garra gotyla (Gray) & Buduna & $\mathrm{C}$ \\
\hline 25. & $\begin{array}{l}\text { Family: Cobitidae } \\
\text { Acanthocobotis botia (Hamilton-Bachanan) }\end{array}$ & Pate Gadela & $\mathrm{PRO}$ \\
\hline 26. & Lepidocephalus guntea (Hamilton-Bachanan) & Lata & CDR \\
\hline 27. & Botia lohachata (Chaudhuri) & Baghi & $\mathrm{UN}$ \\
\hline 28. & $\begin{array}{l}\text { Family: Psilorhynchidae } \\
\text { Psilorhyncus sucatio (Hamilton-Bachanan) }\end{array}$ & Titae & CDR \\
\hline 29. & $\begin{array}{l}\text { IV. ORDER: SILURIFORMES; Family: Bagridae } \\
\text { Mystus cavasius (Hamilton-Bachanan) }\end{array}$ & Tengara & $\mathrm{C}$ \\
\hline & M. bleekeri (Day) & Tengra & $\mathrm{C}$ \\
\hline & M. tengara (Hamilton-Bachanan) & Tengara & $\mathrm{C}$ \\
\hline & M. vittatus (Bloch) & Kanti tengara & $\mathrm{C}$ \\
\hline & $\begin{array}{l}\text { Family: Siluridae } \\
\text { Ompok bimaculatus (Bloch) }\end{array}$ & Papta machha & $\mathrm{C}$ \\
\hline & Wallago attu (Schneider) & Buhari & $\mathrm{C}$ \\
\hline & $\begin{array}{l}\text { Family: Schilbeidae } \\
\text { Clupisoma garua (Hamilton-Bachanan) }\end{array}$ & Jalkapoor & UN \\
\hline 36. & $\begin{array}{l}\text { Family: Clariidae } \\
\text { Clarias batrachus (Linnaeus) }\end{array}$ & Mungri & $\mathrm{C}$ \\
\hline
\end{tabular}




\begin{tabular}{|c|c|c|c|}
\hline 37. & $\begin{array}{l}\text { Family: Heteropneustidae } \\
\text { Heteropneustes fossilis (Bloch) }\end{array}$ & Singhi & $\mathrm{C}$ \\
\hline 38. & $\begin{array}{l}\text { Family: Chacidae } \\
\text { Chaca chaca (Hamilton-Bachanan) }\end{array}$ & Datkitta & $\mathrm{CDR}$ \\
\hline 39. & $\begin{array}{l}\text { V. ORDER: BELONIFORMES; Family: Belonidae } \\
\text { Xenentodon cancila (Hamilton-Bachanan) }\end{array}$ & Kauwai & $\mathrm{C}$ \\
\hline 40. & $\begin{array}{l}\text { VI. ORDER: SYNBRANCHIFORMES; } \\
\text { Family: Synbranchidae } \\
\text { Monopterus cuchia (Hamilton-Bachanan) }\end{array}$ & Anahibam & UN \\
\hline 41. & $\begin{array}{l}\text { Family: Mastacembelidae } \\
\text { Macrognathus aral (Bloch \& Schneider) }\end{array}$ & Gaichi & $\mathrm{C}$ \\
\hline 42. & M. pancalus (Hamilton-Bachanan) & Kathgaichi & $\mathrm{C}$ \\
\hline 43. & Mastacembelus armatus (Lacepale) & Chuche bam & $\mathrm{C}$ \\
\hline 44. & $\begin{array}{l}\text { VII. ORDER: PERCIFORMES: Family: Ambassidae } \\
\text { Chanda nama (Hamilton-Bachanan) }\end{array}$ & Chaner bijuwa & $\mathrm{C}$ \\
\hline 45. & Pseudambasis baculis (Hamilton-Bachanan) & Chanari & $\mathrm{C}$ \\
\hline 46. & $\begin{array}{l}\text { Family: Nandidae } \\
\text { Nandus nandus (Hamilton-Bachanan) }\end{array}$ & Dkhoke & $\mathrm{C}$ \\
\hline 47. & $\begin{array}{l}\text { Family: Gobiidae } \\
\text { Glossogobius giuris (Hamilton-Bachanan) }\end{array}$ & Bhulle machha & $\mathrm{C}$ \\
\hline 48 & $\begin{array}{l}\text { Family: Anabantidae } \\
\text { Anabus testudineus (Bloch) }\end{array}$ & Kabai & $\mathrm{C}$ \\
\hline 49 & $\begin{array}{l}\text { Family: Belontiidae } \\
\text { Colisa faciatus (bloch \& Schneider) }\end{array}$ & Kothari & $\mathrm{C}$ \\
\hline 50 & $\begin{array}{l}\text { Family: Channidae } \\
\text { Channa orientalis (Hamilton-Bachanan) }\end{array}$ & Garai & $\mathrm{C}$ \\
\hline 51 & C. marulius (Hamilton-Bachanan) & Saur & $\mathrm{C}$ \\
\hline
\end{tabular}

Some of the remarkable fish species of the Lohandra river are Notopterus notopterus, Chagunius chagunio, Cirrhinus reba, Labeo dero, L. cabasu, Salmostoma bacaila, Barilius bendelisis, B. barna, Garra gotyla, Crossocheilus latius latius, Acanthocobotis botia, Botia lohachata, Mystus species, Ompok bimaculatus, Wallago attu, Chanda nama, Nandus nandus, Anabus testudineus, Channa orientalis and C. marulius. Some game fishes under high fishing pressure reported in the river are C. chagunio, Catla catla, L. dero, L. rohita, Cirrhinus mrigala, Monopterus cuchia, N. notopterus, Clupisoma garua, $W$. attu and $C$. marulius. Similarly, hill stream fishes of the river are G. gotyla, B. lohachata, C. chagunio, B. barna, Lepidocephalichthys guntea and Psilorhyncus sucatio. Jalkapoor (C. garua), B. bendelisis, B. barna, $C$. marulius, $C$. orientalis and $W$. attu are some preferable and tasty fishes of the river having high demand on the different markets of Biratnagar. Fishes like C. garua and C. marulius, are suitable for fishery development in tras-himalayan areas, particularly Hindukush region (Shrestha, 2008). The important native freshwater fishes of the river are C. garua and Chaca chaca. Few examples of larvivorous fishes in the river are B. barna, B. bendelisis, Danio devario, Puntius sarana, P. ticto, C. marulius, C. nama, Nandus nandus, A. testudineus, Colisa fasciatus and Glossogobius giuris.

The threat status of the fishes are 37 common species, 9 uncommon species, 3 conservation dependent and rare, and one for each pristine rare ornamental species and vulnerable species (Fig. 2). 


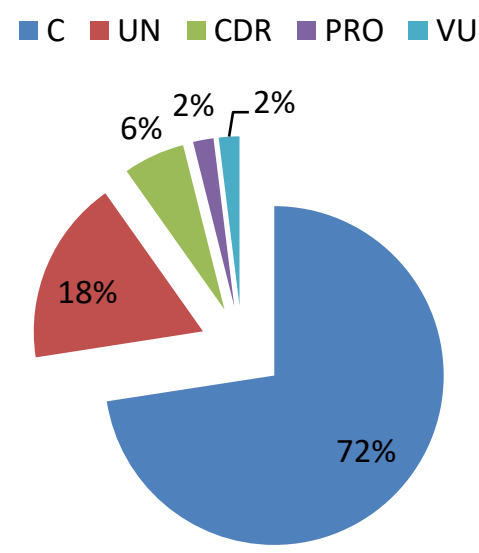

Figure 2. Conservation status (\%) of the species $(\mathrm{C}=$ Common; $\mathrm{UN}=$ Uncommon; $\mathrm{VU}=$ Vulnerable; $\mathrm{PRO}=$ Pristine Rare Ornamental \& $\mathrm{CDR}=$ Conservation Dependent and Rare).

The river has 26 species of Cypriniformes, 10 Siluriformes, 8 Perciformes, 4 Symbranchiformes and one for each of Clupiformes, Osteoglossiformes and Beloniformes (Fig. 3).

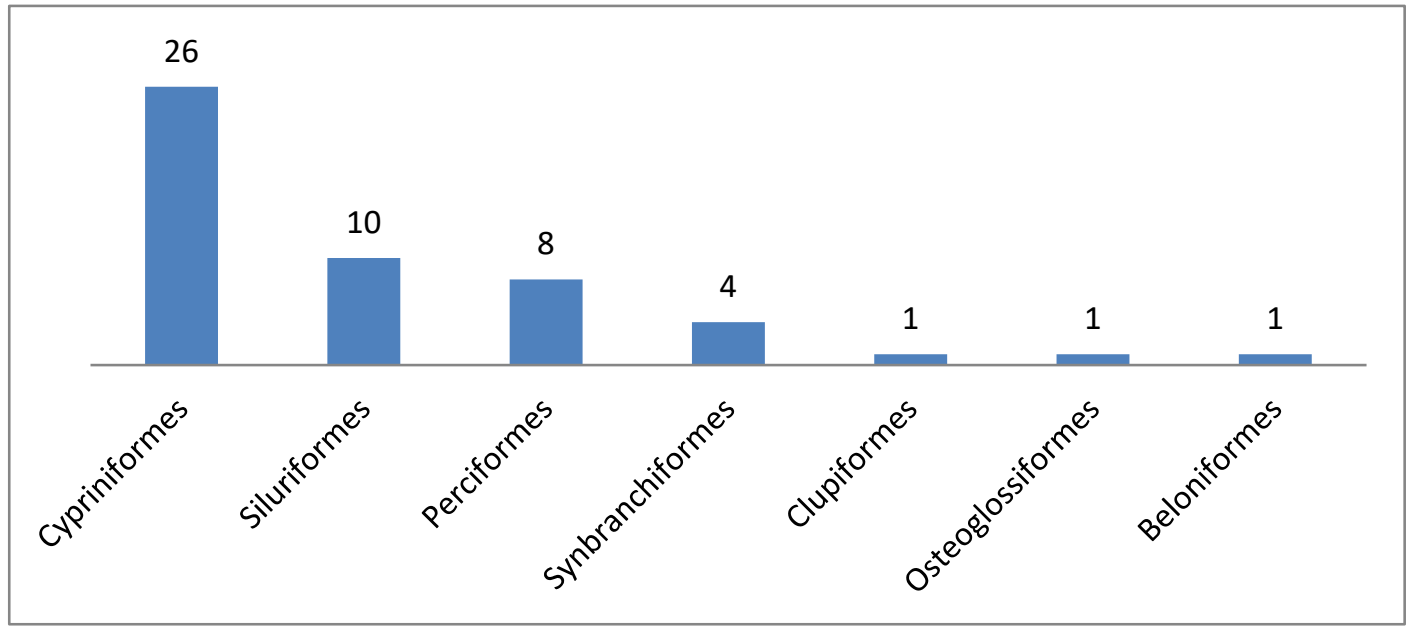

Figure 3. Fish species in different Orders

The occurrence of Cyprinidae in Lohandra river as a dominant species (22) favours the result of Nepal (Shrestha, 2008; Rajbanshi, 2012; Shrestha, 2013). They reported 86 Cyprinidae (Shrestha, 2008), 18 Balitoridae, 12 Bagriidae, 11 Cobitidae, 9 Schilbeidae, 2 Notopteridae, 40 Siroridae, 4 Siluridae and 4 Mastacembelidae. Families like Belonidae, Heteropneustidae, Chacidae and Gobiidae were represented by single species in the present study as well as the report made by Shrestha (2008) and Shrestha (2013). The present report had no any fish species belonging to families Balitoridae, Anguilloidae, Morungidae, Engrulididae, Pangasidae, Amblycipitidae, Sisoridae, Olyridae, Salmonidae, Hemiramphidae, Aplocheilidae, Poeciliidae, Sciaenidae, Cichlidae, Mugidae, Gobioididae 
and Tetraodontidae. The report of fish species made by Shrestha (2013) also includes the fish species found in the present survey.

All fishes reported in Lohandra river in the survey period were also found in Koshi river and Karnali river but $C$. faciatus was not found in Gandaki river and Mahakali river (Rajbanshi, 2012). Fishes A. testudineus, C. faciatus, M. cavasius, M. bleekeri, M. tengara, M. vittatus, X. cancila, C. nama, C. orientalis, C. marulius, G. giuris, O. bimaculatus, Heteropneustes fossilis, Mastacembelus armatus, Clarias batracus, $W$. attu, Puntius sophore, P. sarana, P. ticto, Labeo rohita, L. calbasu, B. lohachata, L. guntea, Aspidoparia jaya, A. morar, C. reba, C. mrigala, Amblypharyngodon mola, D. devario, Chela labuca, Esomus danricus, Crossocheilus latius and C. garua were also reported in Koshi Tappu Wildlife Reserve and some fishes C. chaca, Gadusia chapra, Salmostoma bacaila and $N$. notopterus were described as unavailable fishes after the flood, 2008 (Limbu and Subba, 2011). Fishes like X. cancila, A. botia, B. lohachata, L. guntea, Barilius barna, B. bendelinsis, A. jaya, A. morar, C. reba, C. mrigala, N. notopterus, Pseudambasis baculis, C. nama, C. orientalis, C. marulius, G. giuris, C. chaca, O. bimaculatus, $H$. fossilis, Macrognathus aral, M. puncalus, Mastacembelus armatus, $C$. batracus, $W$. attu, M. cuchia, C. chagunio, C. latius latius, D. devario, $P$. sophore, $P$. sarana, L. rohita and C. faciatus were also mentioned in Koshi river but fishes G. gotyla, G. chapra, C. catla, L. dero, L. calbasu, P. terio, P. ticto, C. labuca, Amblypharyngodon mola, E. danricus, Psilorhyncus sucatio, Mystus cavasius, M. bleekeri, M. tengara, $M$. vittatus, C. garua and A. testudineus were not recorded (Rijal et al., 2014).

Fishes Puntius sophore, P. ticto, P. sarana, S. bacaila, B. bendelisis, B. barna, A. morar, A. mola, C. reba, C. mrigala, C. chagunio, G. gotyla, L. guntea, C. latius latius, $D$. devario, C. garua, C. chaca, M. cuchia, M. cavasius, M. bleekeri, M. tengara, M. vittatus, $O$. bimaculatus, $H$. fossilis, $M$. aral, M. puncalus, M. armatus, C. batracus, W. attu, A. botia, B. lohachata, E. danricus, N. notopterus, G. chapra, C. catla, L. rohita, L. dero, L. calbasu, Pseudambasis baculis, C. nama, Nandus nandus, A. testudineus, C. faciatus, $C$. orientalis, C. marulius, and G. giuris were also reported in Narayani river but fishes $P$. terio, C. labuca, A. jaya, P. sucatio and X. cancila were not reported (Jha and Bhujel, 2014).

The river has also some threatened species like C. chagunio, C. chaca, A. botia and L. guntea. Domestication and captive breeding of some fishes like C. batracus, L. dero, Garra gotyla, B. lohachata and C. chagunio, are being practiced in Fishery Research Centre and Hatchery Centre in different part of the country like C. chagunio, B. lohachata, G. gotyla and $L$. dero (Kaligandaki Fish Hatchery, Syanja and Fisheries Research Centre, Pokhara) and C. batracus (Fisheries Research Centre, Pokhara ). C. chagunio, a table fish, also inhabits in torrential rivers such as Trisuli and Tadi and it is also present in lake Phewa at an altitude of $742 \mathrm{~m}$. Jalkapoor (C. garua), a high demand fish in Biratnagar Markets, is also present in rivers Trisuli, Narayani and Karnali upto 1440 m (Shrestha, 1981).

Lohandra river has great biodiversity especially of ichthyofauna. Present study includes 51 species belonging to 38 genera, 20 families of 7 orders. Some of the remarkable fish species are C. batracus, G. gotyla, B. lohachata, C. chagunio, C. chaca, A. botia, L. guntea, C. faciatus, N. notopterus, C. reba, C. mrigala, L. dero, L. cabasu, S. bacaila, B. bendelisis, B. barna, C. latius latius, Mystus species, O. bimaculatus, W. attu, C. nama, N. nandus, A. testudineus, C. orientalis, C. marulius, C. catla, M. cuchia, C. garua and $P$. 
sucatio. In-depth investigation of ecology and fishes of Lohandra river is recommended for the conservation of these fish species.

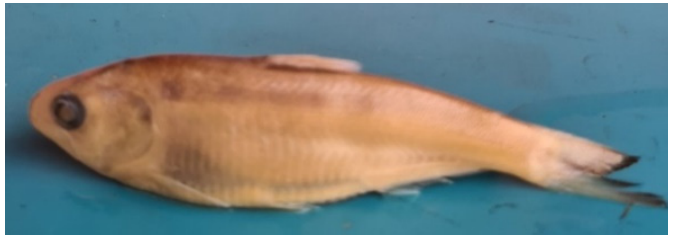

Gadusia chapra

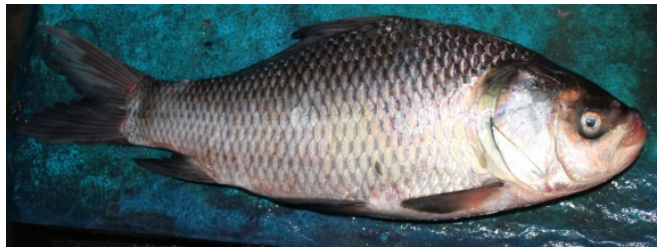

Catla catla

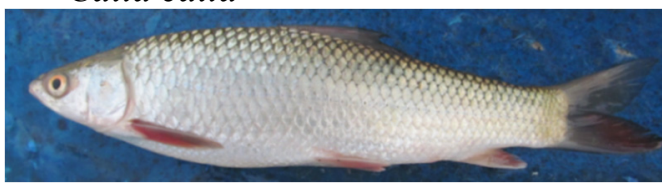

Cirrhinus mrigala

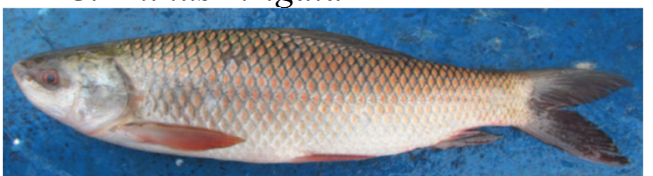

Labeo rohita

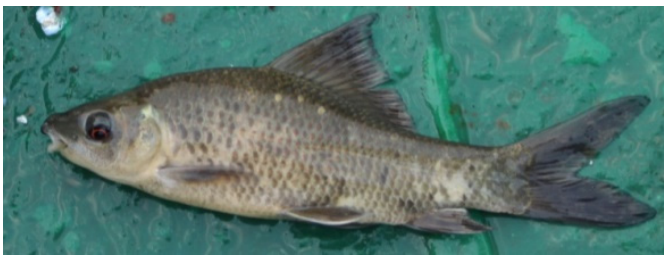

Labeo calbasu

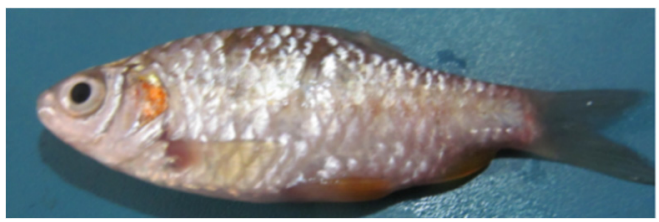

P. sarana

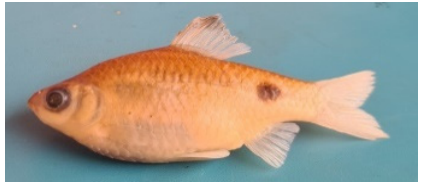

Puntius terio

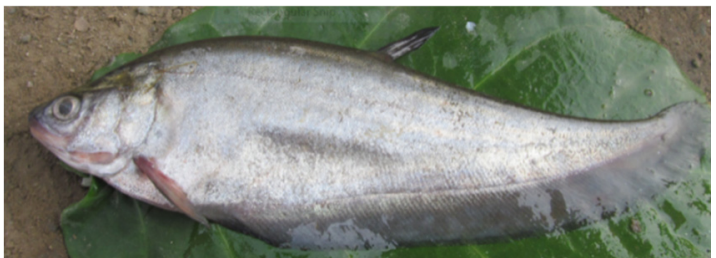

Notopterus notopterus

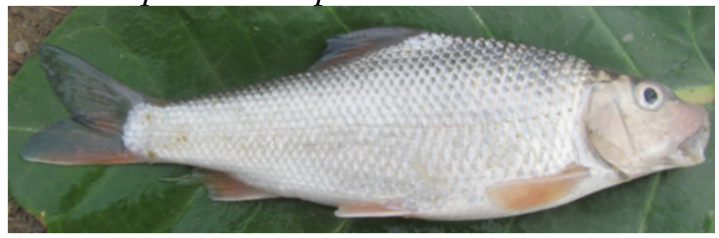

Chagunius chagunio

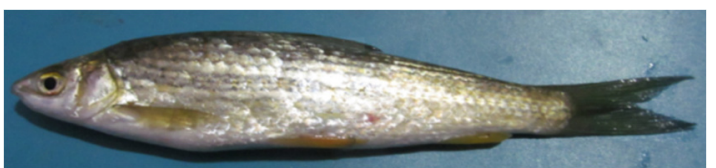

Cirrhinus reba

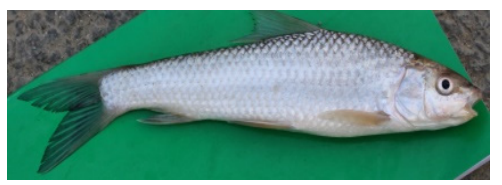

Labeo dero

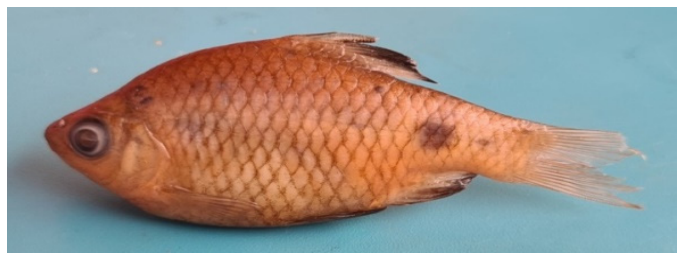

Puntius ticto

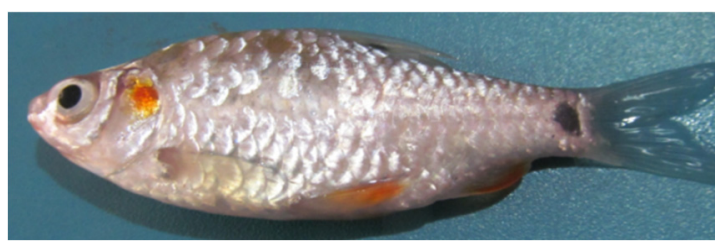

Puntius sophore

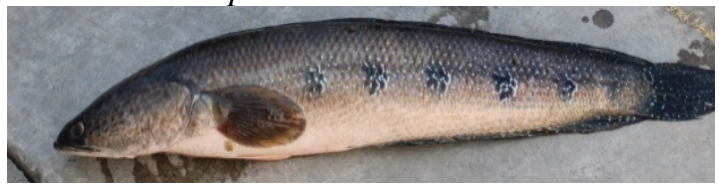

Channa marulius 


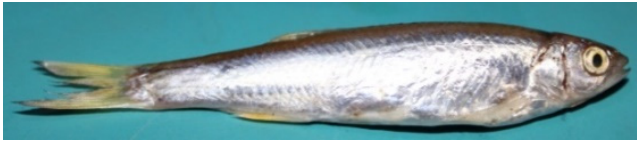

Salmostoma bacaila

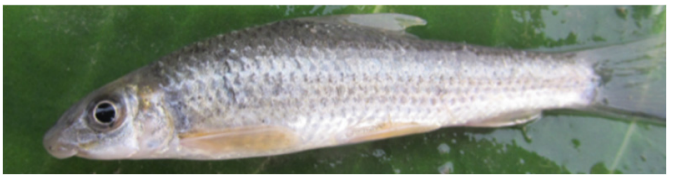

Aspidoparis jaya

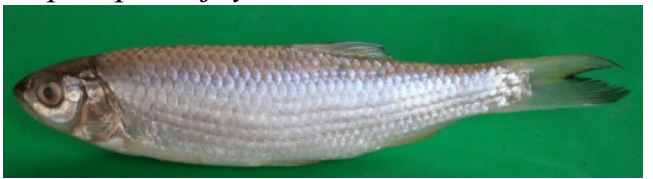

Barilius bendelisis

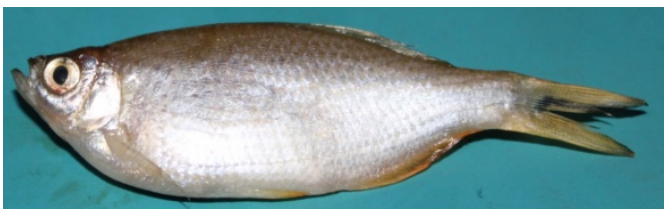

Danio devario

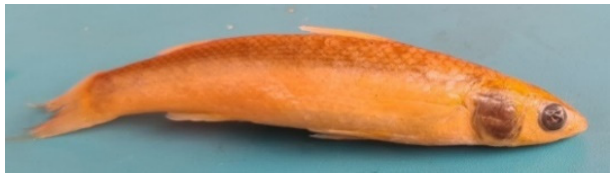

Crossocheilus latius latius

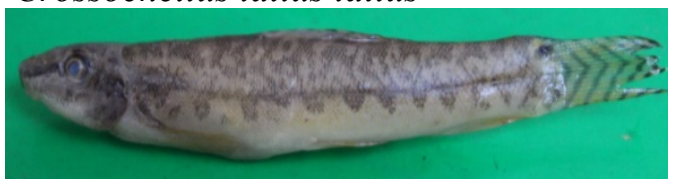

Acanthocobotis botia

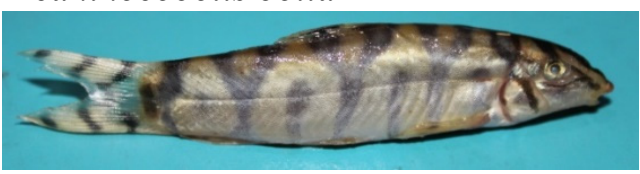

\section{Botia lohachata}

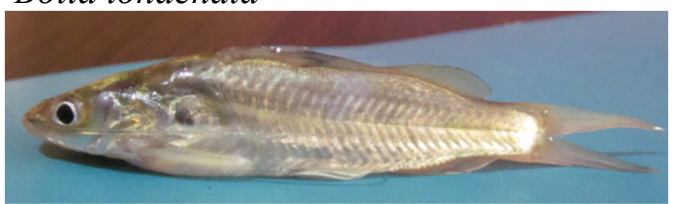

Mystus cavasius

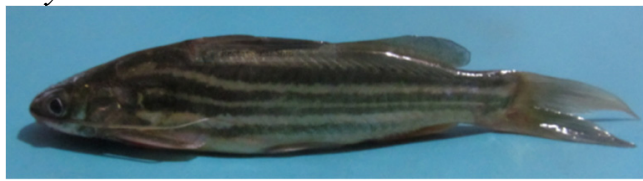

M. tengara

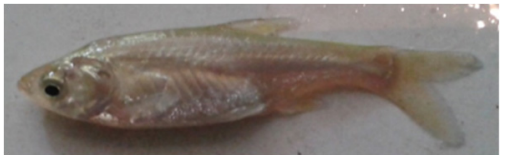

Amblypharyngodon mola
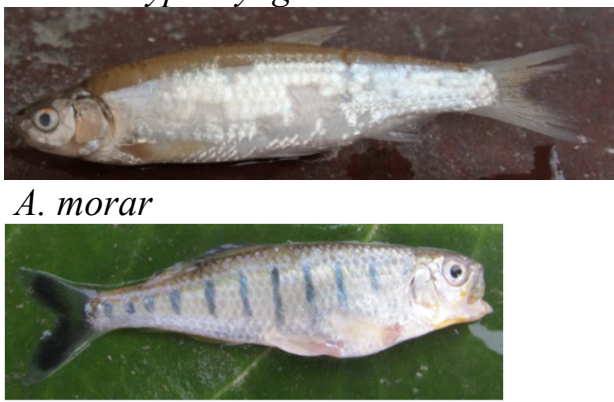

B. barna

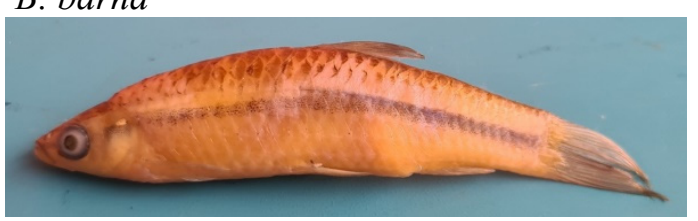

Esomus danricus

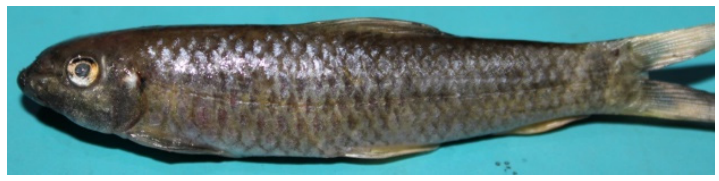

Garra gotyla

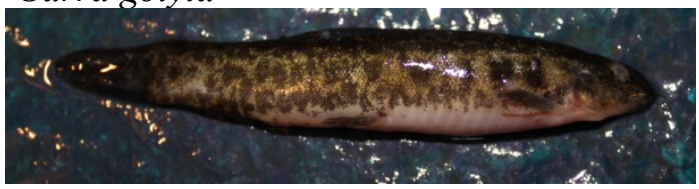

Lepidocephalus guntea

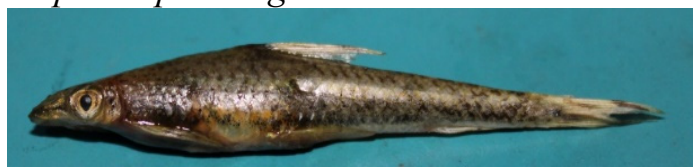

Psilorhyncus sucatio

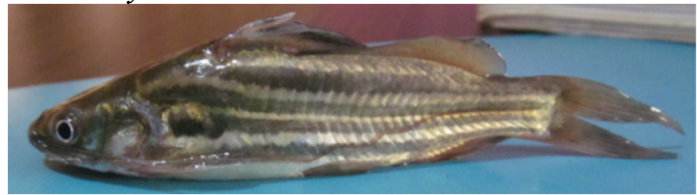

M. bleekeri

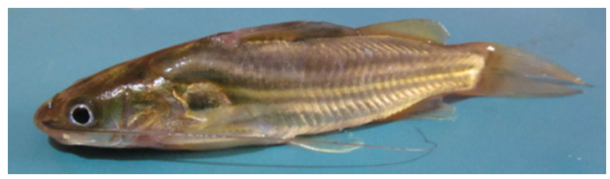

M. vittatus 


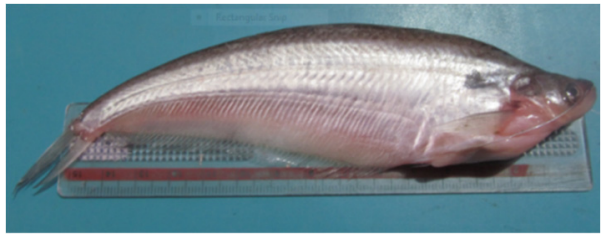

Ompok bimaculatus

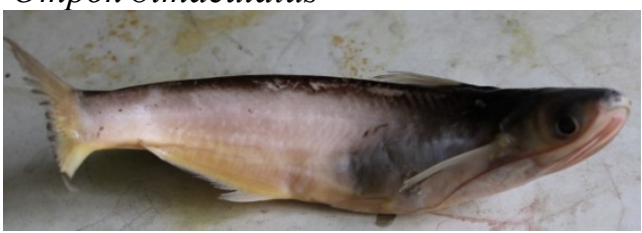

Clupisoma garua

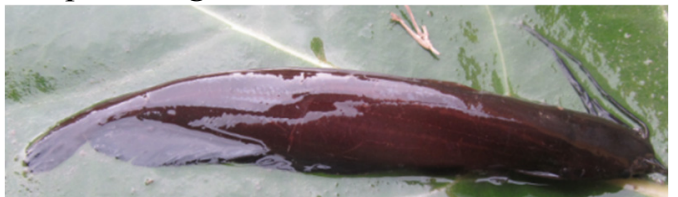

Heteropneustes fossilis

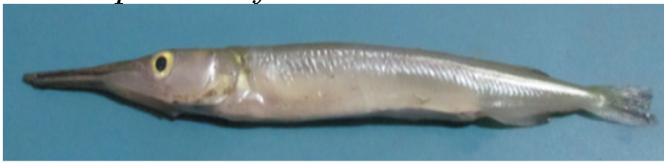

Xenentodon cancila
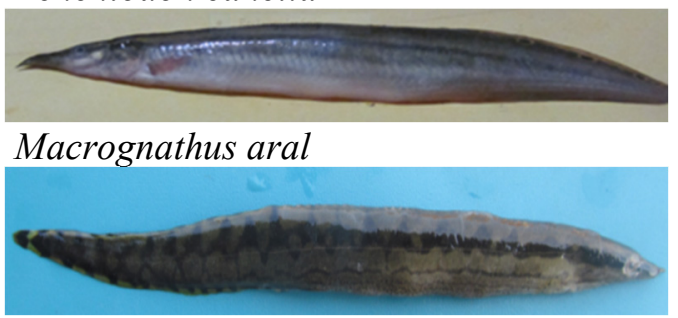

Mastacembelus armatus

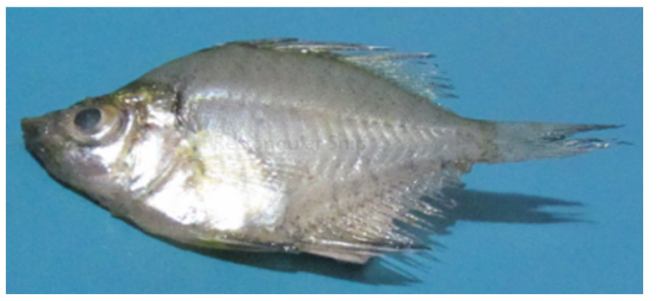

Pseudambasis baculis

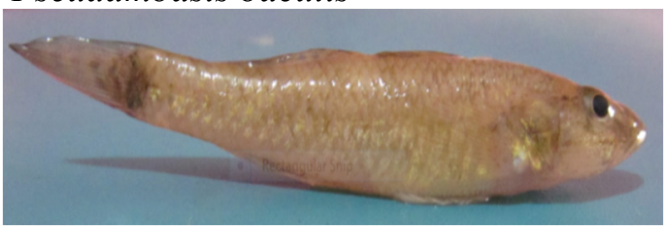

Glossogobius giuris

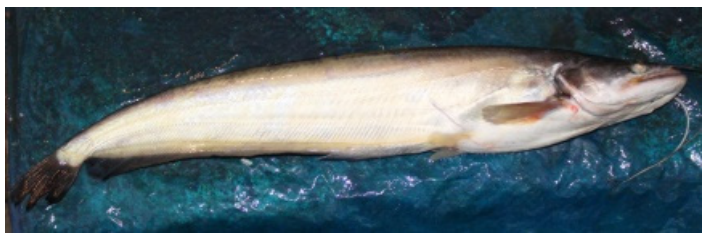

Wallago attu
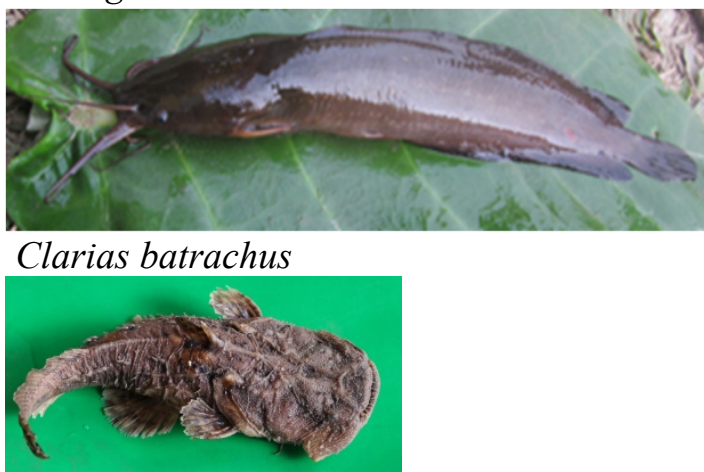

\section{Chaca chaca}

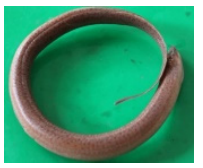

Monopterus cuchia
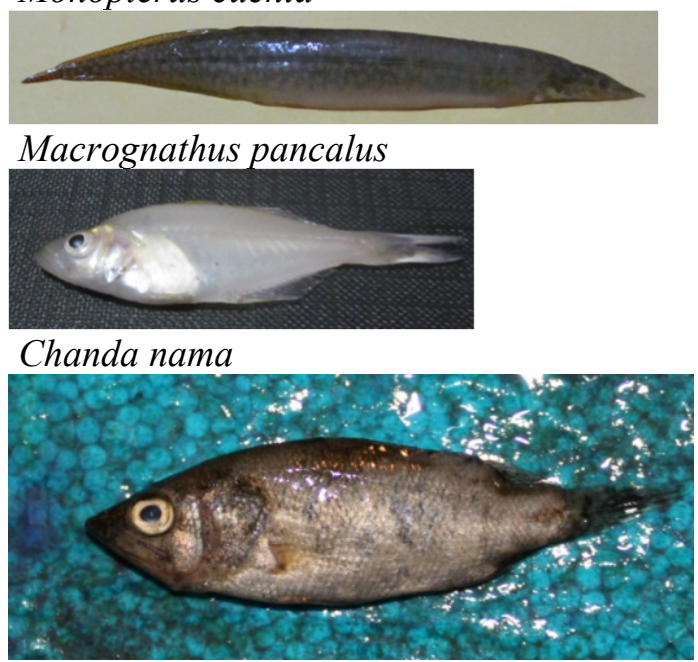

Nandus nandus

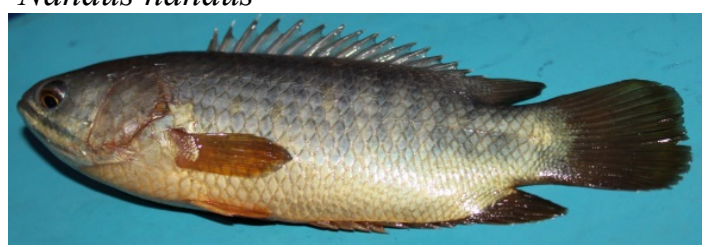

Anabus testudineus 


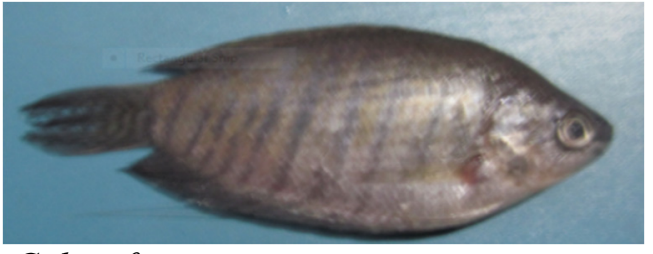

Colisa faciatus

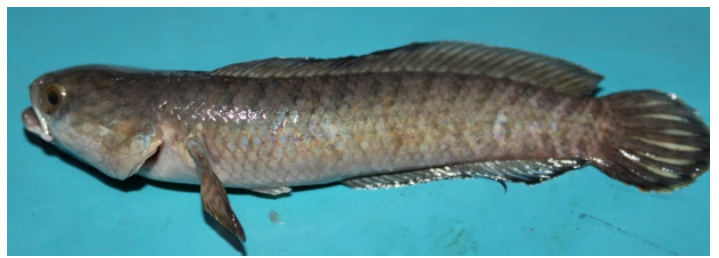

Channa orientalis

Figure 4. Fishes of Lohandra river.

\section{Acknowledgement}

I would like to give a special thanks to Associate Professor, Dr. Bharat Raj Subba, Post Graduate Campus, Tribhuvan University, Biratnagar for his cooperation on identification of the fishes. I would like to express my deep sense of gratitude to Professor Dr. Shiva Kumar Rai, P. G. Campus, T.U., Biratnagar for encouragement and coordination to complete the work. I would give thanks to my parents Late Manamaya Shrestha and Man Bahadur Shrestha as well as Mrs Hari Maya Shrestha for their financial support, without them the work would be incomplete. I sincerely thanks to fisherman Jugasor, who helped us in collection of fishes from the river.

\section{References}

De Witt, H.H. 1960. A contribution to the ichthyology of Nepal. Standford Ichthyological Bulletin, 7(4):63-88.

Edds, D.R. 1986. Fishes of Kali Gandaki / Narayani River, Nepal. Journal of Natural History Museum, 10(14):13-22.

Gupta, S. 2015. Silonia silondia (Hamilton, 1822), A Threatened Fish of Indian Subcontinent. World Journal of Fish and Marine Science, 7(5): 362-364.

Jha, D.K. \& R. C. Bhujel. 2014. Fish Diversity of Narayani River System in Nepal. Nepalese Journal of Aquaculture and Fisheries, 1: 94-108.

Limbu, K.P. \& B.R. Subba. 2011. Status of key faunal in Koshi Tappu Wildlife Reserve after Koshi flood disaster 2008. Nepalese Journal of Biosciences, 1:41-54.

Majumdar, N.N., T.C. Majupuria \& J. Shretha.1972. New records from Nepal. Journal of Zoological Society of India, 2:213-215.

Pokharel, K.K. 2012. Study on Fish Biology of the Seti Gandaki River, Pokhara:11. SpatioTemporal Variation in Fish Communities. Nepal Journal of Science and Technology, 12:350-357.

Rajbanshi, K.G. 2012. Biodiversity and distribution of fresh water fishes of Central/Nepal Himalayan Region. Nepalese Journal of Aquaculture and Fisheries, the Journal of NEFIS. 136p.

Rijal, P.K., M.K. Shrestha, A.K. Singh \& B.R. Dahal. 2014. Fish diversity and their contribution in livelihoods of fishers' in Koshi River basin. Nepalese Journal of Aquaculture, 1: 109-123.

Shrestha, J. 1981. Fishes of Nepal. Curriculum Development Centre, Tribhuvan University, Kathmandu, Nepal. 318p.

Shrestha, J. 1994. Fishes, Fishing implements and Methods of Nepal. Smt. M.D. Gupta, Lalitpur Colony, Lashkar (Gwalior) India (T.C. Majupuria). 150p. 
Shrestha, J. 1998. Aquatic habitats and natural water, fish and fisheries in Nepal. Paper presented in Environmental Assessement Background Training, ADB-TA 2613NEP. NEA-ED, Feb. 2-6, Kathmandu, Nepal. 28p.

Shrestha, J. 2001. Taxonomic Revision of Fishes of Nepal. Environment and Agriculture. In:P.K. jha et al., eds. Biodiversity, Agriculture and Pollution in South Asia, ECOS, Kathmandu:171-180.

Shrestha, J. 2013. Biodiversity: Fish. In: Jha, P.K., Neupane, F.P., Shrestha, M.I. \& Khanal I.P. eds, Biological Diversity and Conservation. Nepal Academy of Science and Technology, Khumaltar, Lalitpur, 69-81.

Shrestha, T.K. 1990. Resource ecology of the Himalayan waters. Curriculum Development Centre, Tribhuvan University, Kathmandu, Nepal. 645p.

Shrestha, T.K. 2008. Ichthyology of Nepal: a study of fishes of the Himalayan waters. Himalayan Ecosphere, Kathmandu. 390p.

Shrivastava, G. 1968. Fishes of eastern U.P., Vishwavidyalaya Prakashan, Vanarasi. 163p. Thapa, R.B. \& K.G. Rajbanshi.1968. Reports on few hill stream fishes of Nepal. Regional Seminar of Ecology of Tropical Highland, UNESCO/HMG, Kathmandu, Nepal. 